\title{
Calix[8]arene Sulfonic Acid Catalyzed Three-Component Reaction for Convenient Synthesis of 3,4-Dihydropyrimidin-2(1H)-ones/thiones under Ultrasonic Irradiation
}

\author{
Lin An, ${ }^{*, a, b}$ Li-li Han, ${ }^{a, b}$ Zu-jian Wang, ${ }^{a}$ Tong-hui Huang, ${ }^{a, b}$ and Hui-dong Zhu ${ }^{a}$ \\ ${ }^{a}$ Department of Pharmacy, Xuzhou Medical College; 209 Tongshan Road, Xuzhou 221004, P. R. China: and ${ }^{b}$ Jiangsu \\ Key Laboratory of New Drug Research and Clinical Pharmacy, Xuzhou Medical College; 209 Tongshan Road, \\ Xuzhou 221004, P. R. China.
}

Received September 3, 2015; accepted November 18, 2015; advance publication released online December 3, 2015

In this work, the catalytic activity of calix[8]arene sulfonic acid was successfully investigated for the famous Biginelli reaction. Under ultrasonic irradiation, calix[8]arene sulfonic acid could efficiently catalyzed the three-component reaction of aldehydes with ethyl acetoacetate and urea or thiourea in ethanol to afford the corresponding 3,4-dihydropyrimidin-2(1H)-ones/thiones in 46-93\%. The advantages of this method are the easy isolated procedure, short reaction time and low cost of the catalyst.

Key words calix[8]arene sulfonic acid; Biginelli reaction; ultrasonic irradiation; 3,4-dihydropyrimidine/thione

Dihydropyrimidinones (DHPMs) and their derivatives are an important class of heterocycles that can be synthesized via Biginelli reaction. ${ }^{1)}$ These heterocycles have gained considerable attention for their pharmacological and biological properties including antiviral, antibacterial behavior, and anticancer activities. $\left.{ }^{2}\right)$ Several derivatives have recently found to be antihypertensive agents, calcium channel blockers, neuropeptide Y(NPY) antagonists and potent HIVgp-120-CD inhibitors. $^{3-6)}$ Hence, numerous methods are known to the synthesis of dihydropyrimidin-2(1H)-ones (DHPMs), including using various Lewis acids as well as protic acids such as xanthan sulfuric acid, ${ }^{7)}$ concentrated $\mathrm{HCl}^{8)} \mathrm{BF}_{3},{ }^{9)}$ polyvinylsulfonic acid, ${ }^{10)}$ $\mathrm{Sm}(\mathrm{OTf})_{3}{ }^{11)} \mathrm{BF}_{3} \cdot \mathrm{OEt}_{2} / \mathrm{CuCl},{ }^{12)}$ and $\mathrm{CeCl}_{3} \cdot 7 \mathrm{H}_{2} \mathrm{O}$. ${ }^{13)}$ However, these methods have several disadvantages, such as unsatisfactory yields, long reaction times, difficult handling of reagents and expensive catalysts. Therefore, chemists continue to focus more attention to find simple, mild and environmental friendly synthetic methods for the DHPMs. Ultrasound has gradually been introduced in organic synthesis as a green-synthetic approach over the last decades. Compared with traditional methods, this technique is more efficient, innocuous, convenient and easily controlled. A large number of organic reactions, including Biginelli reactions, can be efficiently carried out with high yields under ultrasonic irradiation. ${ }^{14-17)}$

Based on our research on the development of useful synthetic methodologies, ${ }^{18)}$ we successfully prepared 4,6diaryl-3,4-dihydro-pyrimidin-2(1H)-ones which were catalyzed by $\left(\mathrm{CH}_{3}\right)_{3} \mathrm{SiCl}$. Recently, calix[4]arene sulfonic acid has displayed potential and effective applications as catalyst in organic reactions, ${ }^{19-24)}$ including Mannich-type reactions, alkylation, esterification, nucleophilic substitution and Biginelli reaction. However, calix[8]arene sulfonic acid, which has the bigger cavity size than that of calix[4]arene sulfonic acid, has never been reported as catalyst in Biginelli reaction. To continue our interest in Lewis acid catalyst applications for DHPMs and ultrasonic irradiation-assisted synthesis, we report a three-component reaction of aldehydes with ethyl acetoacetate and urea or thiourea in ethanol catalyzed by calix[8]- arene sulfonic acid under ultrasonic irradiation.

\section{MATERIALS AND METHODS}

Reagents and Apparatus Melting points were determined with capillaries with an YRT-3 microscope apparatus and were uncorrected. ${ }^{1} \mathrm{H}-\mathrm{NMR}$ spectra were recorded at $600 \mathrm{MHz}$ on a Bruker AV-600 spectrometer. IR spectra were obtained on a Nicolet Fourier transform (FT)-IR 8400 spectrometer $(\mathrm{KBr}$ disc). All reagents and solvents were commercial reagents with analytical grade. Calix[8]arene sulfonic acid was prepared according to the published method. ${ }^{25)}$ Reactions were monitored by TLC on $2.5 \mathrm{~mm}$ Merck silica gel F254 strips.

General Procedure for the Synthesis of 3,4-Dihydropyrimidin-2(1H)-ones/thiones $4 \mathbf{a}-\mathbf{p}$ A mixture of aldehyde $(1.0 \mathrm{mmol})$, ethyl acetoacetate $(1.0 \mathrm{mmol})$ and urea or thiourea $(1.5 \mathrm{mmol})$ in refluxing ethanol $(3.0 \mathrm{~mL})$ contained calix[8]arene sulfonic acid $(0.2 \mathrm{~mol} \%)$ was assisted by ultrasonic irradiation for a specified period. Reactions were monitored by TLC. Then, the mixtures were allowed to be added into cooled water, the solid was filtered, and washed with few cold water, ethanol, and dried under vacuum to give the pure product.

\section{Spectral Data for $\mathbf{4 a - p}$}

Ethyl 4-(4-Chlorophenyl)-6-methyl-2-oxo-1,2,3,4-tetrahydropyrimidine-5-carboxylate (4a)

White powder. ${ }^{1} \mathrm{H}-\mathrm{NMR}$ (dimethyl sulfoxide (DMSO)- $d_{6}$ ) $\delta: 1.09(3 \mathrm{H}, \mathrm{t}, J=7.2 \mathrm{~Hz}), 2.25(3 \mathrm{H}, \mathrm{s}), 3.99(2 \mathrm{H}, \mathrm{q}, J=7.2 \mathrm{~Hz})$, $5.14(1 \mathrm{H}, \mathrm{s}), 7.25(2 \mathrm{H}, \mathrm{d}, J=8.4 \mathrm{~Hz}), 7.39(2 \mathrm{H}, \mathrm{d}, J=8.4 \mathrm{~Hz})$, $7.73(1 \mathrm{H}, \mathrm{s}), 9.20(1 \mathrm{H}, \mathrm{s})$. IR $(\mathrm{KBr}) \mathrm{cm}^{-1}: 3235,3115,2981$, 1717, 1695, 1651, 1495, 1460, 1288, 1227, 1091, 958, 788.

Ethyl 6-Methyl-4-( $p$-tolyl)-2-oxo-1,2,3,4-tetrahydropyrimidine5-carboxylate (4b)

White powder. ${ }^{1} \mathrm{H}-\mathrm{NMR}$ (DMSO- $\left.d_{6}\right) \quad \delta: 1.10 \quad(3 \mathrm{H}, \mathrm{t}$, $J=7.2 \mathrm{~Hz}), 2.23(3 \mathrm{H}, \mathrm{s}), 2.26(3 \mathrm{H}, \mathrm{s}), 3.98(2 \mathrm{H}, \mathrm{q}, J=7.2 \mathrm{~Hz})$, $5.10(1 \mathrm{H}, \mathrm{s}), 7.11(4 \mathrm{H}, \mathrm{s}), 7.64(1 \mathrm{H}, \mathrm{s}), 9.10(1 \mathrm{H}, \mathrm{s})$. IR $(\mathrm{KBr})$ $\mathrm{cm}^{-1}: 3242,3122,2981,1724,1705,1651,1495,1460,1402$, 
1288, 1228, 1090, 787.

Ethyl 4-(4-(tert-Butyl)phenyl)-6-methyl-2-oxo-1,2,3,4-tetrahydropyrimidine-5-carboxylate $(4 \mathrm{c})$

White powder. ${ }^{1} \mathrm{H}-\mathrm{NMR}$ (DMSO- $\left.d_{6}\right) \quad \delta: 1.12(3 \mathrm{H}, \mathrm{t}$, $J=7.2 \mathrm{~Hz}), 1.25(9 \mathrm{H}, \mathrm{s}), 2.23(3 \mathrm{H}, \mathrm{s}), 3.99(2 \mathrm{H}, \mathrm{q}, J=7.2 \mathrm{~Hz})$, $5.11(1 \mathrm{H}, \mathrm{s}), 7.15(2 \mathrm{H}, \mathrm{d}, J=7.8 \mathrm{~Hz}), 7.33(2 \mathrm{H}, \mathrm{d}, J=8.4 \mathrm{~Hz}), 7.64$ $(1 \mathrm{H}, \mathrm{s}), 9.12(1 \mathrm{H}, \mathrm{s}) .{ }^{13} \mathrm{C}-\mathrm{NMR}$ (DMSO- $\left.d_{6}\right) \delta: 14.6,18.3,31.6$, $34.6,39.4,39.6,40,40.2,40.4,40.6,53.9,59.7,100,125.6$, $126.4,142.4,148.6,150.1,152.8,165.9$. IR (KBr) cm $\mathrm{cm}^{-1}: 3354$, 3219, 3111, 2961, 2879, 1724, 1693, 1643, 1495, 1456, 1402, 1227, 1097, 787, 658. Electrospray ionization-high resolution (ESI-HR)-MS Calcd for $\mathrm{C}_{18} \mathrm{H}_{24} \mathrm{~N}_{2} \mathrm{O}_{3}[\mathrm{M}+\mathrm{Na}]^{+}$339.1685. Found 339.1691.

Ethyl 4-(2-Methoxyphenyl)-6-methyl-oxo-1,2,3,4-tetrahydropyrimidine-5-carboxylate (4d)

White powder. ${ }^{1} \mathrm{H}-\mathrm{NMR}\left(\mathrm{DMSO}-d_{6}\right) \delta: 1.02(3 \mathrm{H}, \mathrm{t}$, $J=7.2 \mathrm{~Hz}), 2.28(3 \mathrm{H}, \mathrm{s}), 3.79(3 \mathrm{H}, \mathrm{s}), 3.92(2 \mathrm{H}, \mathrm{q}, J=6.6 \mathrm{~Hz})$, $5.49(1 \mathrm{H}, \mathrm{s}), 6.87(1 \mathrm{H}, \mathrm{t}, J=7.8 \mathrm{~Hz}), 6.98(1 \mathrm{H}, \mathrm{d}, J=8.4 \mathrm{~Hz})$, $7.05(1 \mathrm{H}, \mathrm{d}, J=7.8 \mathrm{~Hz}), 7.21(1 \mathrm{H}, \mathrm{s}), 7.23(1 \mathrm{H}, \mathrm{d}, J=7.8 \mathrm{~Hz})$, 9.07 (1H, s). IR (KBr) cm $\mathrm{cm}^{-1}: 3242,3125,2989,1726,1701$, 1637, 1487, 1402, 1286, 1215, 1080, 787, 763.

Ethyl 4-(4-Hydroxyphenyl)-6-methyl-2-oxo-1,2,3,4-tetrahydropyrimidine-5-carboxylate (4e)

White powder. ${ }^{1} \mathrm{H}-\mathrm{NMR}$ (DMSO- $\left.d_{6}\right) \quad \delta: 1.10 \quad(3 \mathrm{H}, \mathrm{t}$, $J=7.2 \mathrm{~Hz}), 2.23(3 \mathrm{H}, \mathrm{s}), 3.98(2 \mathrm{H}, \mathrm{q}, J=7.2 \mathrm{~Hz}), 5.04(1 \mathrm{H}, \mathrm{s})$, $6.68(2 \mathrm{H}, \mathrm{d}, J=7.8 \mathrm{~Hz}), 7.02(2 \mathrm{H}, \mathrm{d}, J=7.8 \mathrm{~Hz}), 7.57(1 \mathrm{H}, \mathrm{s})$, $9.06(1 \mathrm{H}, \mathrm{s}), 9.28(1 \mathrm{H}, \mathrm{s}) . \mathrm{IR}(\mathrm{KBr}) \mathrm{cm}^{-1}: 3284,1717,1686$, $1655,1510,1400,1229,1097,812$.

Ethyl 4-(4-Fluorophenyl)-6-methyl-2-oxo-1,2,3,4-tetrahydropyrimidine-5-carboxylate (4f)

White powder. ${ }^{1} \mathrm{H}-\mathrm{NMR}$ (DMSO- $\left.d_{6}\right) \quad \delta: 1.09(3 \mathrm{H}, \mathrm{t}$, $J=7.2 \mathrm{~Hz}), 2.25(3 \mathrm{H}, \mathrm{s}), 3.98(2 \mathrm{H}, \mathrm{q}, J=6.6 \mathrm{~Hz}), 5.14(1 \mathrm{H}, \mathrm{s})$, $7.14(2 \mathrm{H}, \mathrm{t}, J=6.6 \mathrm{~Hz}), 7.26(2 \mathrm{H}, \mathrm{t}, J=8.4 \mathrm{~Hz}), 7.71(1 \mathrm{H}, \mathrm{s}), 9.18$ $(1 \mathrm{H}, \mathrm{s})$. IR $(\mathrm{KBr}) \mathrm{cm}^{-1}: 3223,3113,2981,1717,1685,1653$, 1599, 1514, 1448, 1288, 1227, 1169, 1097, 958, 798.

Ethyl 6-Methyl-2-oxo-4-(m-toyl)-1,2,3,4-tetrahydropyrimidine-5-carboxylate (4g)

White powder. ${ }^{1} \mathrm{H}-\mathrm{NMR}$ (DMSO- $\left.d_{6}\right) \quad \delta: 1.10 \quad(3 \mathrm{H}, \mathrm{t}$, $J=7.2 \mathrm{~Hz}), 2.24(3 \mathrm{H}, \mathrm{s}), 2.28(3 \mathrm{H}, \mathrm{s}), 3.98-4.00(2 \mathrm{H}, \mathrm{m}), 5.11$ $(1 \mathrm{H}, \mathrm{s}), 7.04(3 \mathrm{H}, \mathrm{q}, J=7.2 \mathrm{~Hz}), 7.20(1 \mathrm{H}, \mathrm{t}, J=7.2 \mathrm{~Hz}), 7.65$ $(1 \mathrm{H}, \mathrm{s}), 9.11(1 \mathrm{H}, \mathrm{s}) . \mathrm{IR}(\mathrm{KBr}) \mathrm{cm}^{-1}: 3231,3120,2991,2927$, 1705, 1651, 1427, 1224, 1228, 1090, 1024, 767.

Ethyl 4-(4-Bromophenyl)-6-methyl-2-oxo-1,2,3,4-tetrahydropyrimidine-5-carboxylate (4h)

White powder. ${ }^{1} \mathrm{H}-\mathrm{NMR}$ (DMSO- $\left.d_{6}\right) \delta: 1.10 \quad(3 \mathrm{H}, \mathrm{t}$, $J=7.2 \mathrm{~Hz}), 2.25(3 \mathrm{H}, \mathrm{s}), 3.99(2 \mathrm{H}, \mathrm{q}, J=7.2 \mathrm{~Hz}), 5.12(1 \mathrm{H}, \mathrm{s})$, $7.19(2 \mathrm{H}, \mathrm{d}, J=7.8 \mathrm{~Hz}), 7.52(2 \mathrm{H}, \mathrm{d}, J=8.4 \mathrm{~Hz}), 7.73(1 \mathrm{H}, \mathrm{s})$, $9.20(1 \mathrm{H}, \mathrm{s})$. IR $(\mathrm{KBr}) \mathrm{cm}^{-1}: 3124,2989,1726,1701,1637$, 1487, 1402, 1286, 1242, 1080, 968, 764.

Ethyl 6-Methyl-4-(3-nitrophenyl)-2-oxo-1,2,3,4-tetrahydropyrimidine-5-carboxylate (4i)

Pale yellow powder. ${ }^{1} \mathrm{H}-\mathrm{NMR}$ (DMSO- $\left.d_{6}\right) \delta: 1.13(3 \mathrm{H}, \mathrm{t}$, $J=7.2 \mathrm{~Hz}), 2.25(3 \mathrm{H}, \mathrm{s}), 3.99(1 \mathrm{H}, \mathrm{q}, J=7.2 \mathrm{~Hz}), 5.05(1 \mathrm{H}, \mathrm{s})$, $7.77(1 \mathrm{H}, \mathrm{s}), 8.06-8.25(4 \mathrm{H}, \mathrm{m}), 9.35(1 \mathrm{H}, \mathrm{s}) . \mathrm{IR}(\mathrm{KBr}) \mathrm{cm}^{-1}$ : $3458,3308,3244,3121,1728,1647,1607,1520,1350,1217$, 1088, 1026, 956, 872, 783.

Ethyl 6-Methyl-4-(4-nitrophenyl)-2-oxo-1,2,3,4-tetrahydropyrimidine-5-carboxylate $(\mathbf{4 j})$

Pale yellow powder. ${ }^{1} \mathrm{H}-\mathrm{NMR}$ (DMSO- $\left.d_{6}\right) \delta$ : $1.17(3 \mathrm{H}, \mathrm{t}$, $J=7.2 \mathrm{~Hz}), 2.27(3 \mathrm{H}, \mathrm{s}), 3.99(2 \mathrm{H}, \mathrm{q}, J=7.2 \mathrm{~Hz}), 5.28(1 \mathrm{H}, \mathrm{s})$, $7.86(1 \mathrm{H}, \mathrm{s}), 8.16-8.23(4 \mathrm{H}, \mathrm{m}), 9.32(1 \mathrm{H}, \mathrm{s}) . \mathrm{IR}(\mathrm{KBr}) \mathrm{cm}^{-1}$ :

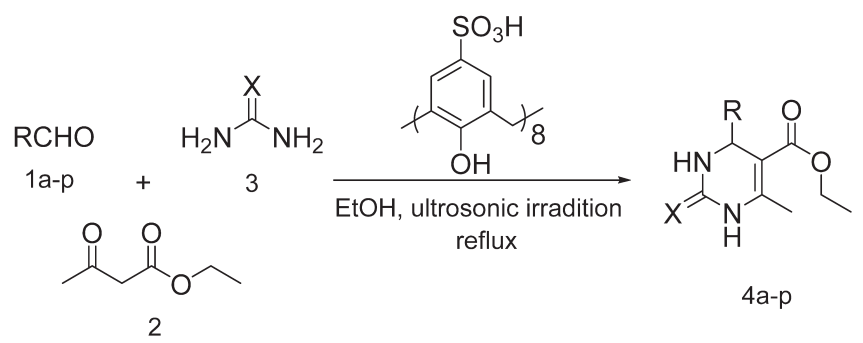

Chart 1. Calix[8]arene Sulfonic Acid Catalyzed Biginelli Reactions

3441, 3337, 3296, 1727, 1666, 1610, 1477, 1282, 1202, 1093, 923, 734 .

Ethyl 4-Ethyl-6-methyl-2-oxo-1,2,3,4-tetrahydropyrimidine5-carboxylate (4k)

White powder. ${ }^{1} \mathrm{H}-\mathrm{NMR}$ (DMSO- $\left.d_{6}\right) \quad \delta: 0.81 \quad(3 \mathrm{H}, \mathrm{t}$, $J=7.2 \mathrm{~Hz}), 1.20(3 \mathrm{H}, \mathrm{t}, J=7.2 \mathrm{~Hz}), 1.41-1.47(2 \mathrm{H}, \mathrm{m}), 2.19(3 \mathrm{H}$, s), 4.02-4.14 (3H, m), $7.28(1 \mathrm{H}, \mathrm{s}), 8.91(1 \mathrm{H}, \mathrm{s})$. IR $(\mathrm{KBr}) \mathrm{cm}^{-1}$ : $3246,3121,2961,1724,1703,1674,1645,1468,1286,1117$, 1024, 789, 777.

Ethyl 6-Methyl-2-oxo-4-propyl-1,2,3,4-tetrahydropyrimidine5-carboxylate (4I)

White powder. ${ }^{1} \mathrm{H}-\mathrm{NMR}$ (DMSO- $\left.d_{6}\right) \quad \delta: 0.85 \quad(3 \mathrm{H}, \mathrm{t}$, $J=7.2 \mathrm{~Hz}), 1.19(3 \mathrm{H}, \mathrm{t}, J=7.2 \mathrm{~Hz}), 1.23-1.46(4 \mathrm{H}, \mathrm{m}), 2.17(3 \mathrm{H}$, s), 4.00-4.13 (3H, m), $7.31(1 \mathrm{H}, \mathrm{s}), 8.92(1 \mathrm{H}, \mathrm{s})$. IR $(\mathrm{KBr})$ $\mathrm{cm}^{-1}: 3250,3119,2959,1718,1703,1672,1647,1431,1236$, 1092, 1018, 789, 779 .

Ethyl 6-Methyl-2-oxo-4-styryl-1,2,3,4-tetrahydropyrimidine5-carboxylate (4m)

White powder. ${ }^{1} \mathrm{H}-\mathrm{NMR}$ (DMSO- $\left.d_{6}\right) \quad \delta: 1.20 \quad(3 \mathrm{H}, \mathrm{t}$, $J=7.2 \mathrm{~Hz}), 2.20(3 \mathrm{H}, \mathrm{s}), 4.09(2 \mathrm{H}, \mathrm{q}, J=7.2 \mathrm{~Hz}), 4.74(1 \mathrm{H}, \mathrm{s})$, $5.14(1 \mathrm{H}, \mathrm{s}), 5.56(1 \mathrm{H}, \mathrm{s}), 7.24-7.40(5 \mathrm{H}, \mathrm{m}), 7.54(1 \mathrm{H}, \mathrm{s}), 9.14$ $(1 \mathrm{H}, \mathrm{s})$. IR $(\mathrm{KBr}) \mathrm{cm}^{-1}: 3415,3244,1722,1701,1686,1653$, 1419, 1229, 1097,719, 692.

Ethyl 6-Methyl-4-phenyl-2-thioxo-1,2,3,4-tetrahydropyrimidine5-carboxylate (4n)

White powder. ${ }^{1} \mathrm{H}-\mathrm{NMR}$ (DMSO- $\left.d_{6}\right) \quad \delta: 1.11 \quad(3 \mathrm{H}, \mathrm{t}$, $J=7.2 \mathrm{~Hz}), 2.30(3 \mathrm{H}, \mathrm{s}), 4.02(2 \mathrm{H}, \mathrm{q}, J=7.2 \mathrm{~Hz}), 5.18(1 \mathrm{H}, \mathrm{d}$, $J=4.0 \mathrm{~Hz}), 7.22-7.37(5 \mathrm{H}, \mathrm{m}), 9.66(1 \mathrm{H}, \mathrm{s}), 10.34(1 \mathrm{H}, \mathrm{s}) . \mathrm{IR}$ $(\mathrm{KBr}) \mathrm{cm}^{-1}: 3329,3175,1701,1670,1626,1466,1196,1128$, 1028, 694.

Ethyl 6-Methyl-4-(4-methoxyphenyl)-2-thioxo-1,2,3,4-tetrahydropyrimidine-5-carboxylate (4o)

White powder. ${ }^{1} \mathrm{H}-\mathrm{NMR}$ (DMSO- $\left.d_{6}\right) \quad \delta: 1.11 \quad(3 \mathrm{H}, \mathrm{t}$, $J=7.2 \mathrm{~Hz}), 2.29(3 \mathrm{H}, \mathrm{s}), 3.73(3 \mathrm{H}, \mathrm{s}), 4.01(2 \mathrm{H}, \mathrm{q}, J=7.2 \mathrm{~Hz})$, $5.12(1 \mathrm{H}, \mathrm{d}, J=3.6 \mathrm{~Hz}), 6.89-6.92(2 \mathrm{H}, \mathrm{m}), 7.11-7.15(2 \mathrm{H}, \mathrm{m})$, $9.60(1 \mathrm{H}, \mathrm{s}), 10.29(1 \mathrm{H}, \mathrm{s}) . \mathrm{IR}(\mathrm{KBr}) \mathrm{cm}^{-1}: 3315,3171,1663$, 1651, 1610, 1466, 1284, 1196, 1027, 765.

Ethyl 6-Methyl-4-styryl-2-thioxo-1,2,3,4-tetrahydropyrimidine5-carboxylate (4p)

white powder. ${ }^{1} \mathrm{H}-\mathrm{NMR}\left(\mathrm{DMSO}-d_{6}\right) \quad \delta: 1.20 \quad(3 \mathrm{H}, \mathrm{t}$, $J=7.2 \mathrm{~Hz}), 2.25(3 \mathrm{H}, \mathrm{s}), 4.04-4.17(2 \mathrm{H}, \mathrm{m}), 4.76-4.78(1 \mathrm{H}, \mathrm{m})$, $6.18(1 \mathrm{H}, \mathrm{dd}, J=6.4 \mathrm{~Hz}), 6.36(1 \mathrm{H}, \mathrm{d}, J=6.4 \mathrm{~Hz}), 7.23-7.43(5 \mathrm{H}$, m), $9.48(1 \mathrm{H}, \mathrm{s}), 10.31(1 \mathrm{H}, \mathrm{s}) . \mathrm{IR}(\mathrm{KBr}) \mathrm{cm}^{-1}: 3404,3159$, 1707, 1680, 1650, 1593, 1466, 1194, 1109, 968, 754.

\section{RESULTS AND DISCUSSION}

The synthetic route and the molecular structure of calix[8]arene sulfonic acid were depicted in Chart 1.

To the best of our knowledge, ultrasonic irradiation plays 
an important role in the acceleration of the organic reaction rate. ${ }^{26)}$ Thus, we initially investigated the effect of ultrasonic irradiation on the model reaction of 4-chlorobenzaldehyde, ethyl acetoacetate and urea in refluxing ethanol. The experimental results (Table 1) showed that the traditional heating reaction can be finished in eight hours, whereas the reaction time was reduced to $25 \mathrm{~min}$ under ultrasonic irradiation condition and the yield of the product $\mathbf{4 a}$ are higher. Based on the result of this study, ultrasonic irradiation condition was selected. To determine the catalytic efficiency difference between calix $[n]$ arene sulfonic acids and $p$-toluenesulfonic acid, we examined this model reaction using different sulfonic acids which contained an equimolar amount of "acid." To our satisfaction, calix $[n]$ arenes were more efficient than $p$-toluene sulfonic acid (Table 1), which indicated that the sulfonyl and phenolic groups in calixarene structures were responsible for the good yields. Overall, calix[8]arene sulfonic acid was the most efficient catalyst, which was attributed to the presence of more sulfonic acid groups in its structure compared with the other acids.

To optimize reaction conditions, we discuss the effects of catalyst and molar ratio of reagents. The model reactions of 4-chloro benzaldehyde, ethyl acetoacetate and urea with various amounts of calix[8]arene sulfonic acid were investigated (Table 2). To determine the role of a catalyst, we conducted a blank reaction test in the absence of calix[8]arene sulfonic

Table 1. Comparison of Efficiency of Various Sulfonic Acid Catalysts on the Yields of 4 a under Certain Conditions ${ }^{a)}$

\begin{tabular}{clccc}
\hline \hline No. & \multicolumn{1}{c}{ Catalyst } & Catalyst (mol \%) & Time & Yield of 4a (\%) \\
\hline 1 & Calix[8]arene sulfonic acid & 0.5 & $8 \mathrm{~h}^{b)}$ & 56 \\
2 & Calix[8]arene sulfonic acid & 0.5 & $25 \mathrm{~min}^{c)}$ & 76 \\
3 & Calix[6]arene sulfonic acid & 0.67 & $28 \mathrm{~min}^{c)}$ & 45 \\
4 & Calix[4]arene sulfonic acid & 1 & $30 \min ^{c)}$ & 37 \\
5 & $p$-Toluenesulfonic acid & 4 & $60 \min ^{c)}$ & 28 \\
\hline
\end{tabular}

a) Reaction conditions: 4-chlorobenzaldehyde $(1.0 \mathrm{mmol})$, ethyl acetoacetate $(1.2 \mathrm{mmol})$, urea $(1.5 \mathrm{mmol})$ in refluxing ethanol $(3.0 \mathrm{~mL}) . b)$ Without ultrasonic irradiation. c) Ultrasonic irradiation

Table 2. Effect of Amount of Calix[8]arene Sulfonic Acid on the Yield of $\mathbf{4 a}$ under Certain Conditions ${ }^{a)}$

\begin{tabular}{cccc}
\hline \hline No. & Catalyst (mol \%) & Reaction time (min) & Yield of 4a (\%) \\
\hline 1 & 0 & 60 & Trace \\
2 & 0.1 & 40 & 35 \\
3 & 0.15 & 35 & 60 \\
4 & 0.2 & 20 & 90 \\
5 & 0.3 & 20 & 88 \\
6 & 0.5 & 22 & 87 \\
7 & 1 & 20 & 85 \\
\hline
\end{tabular}

a) Reaction conditions: 4-chlorobenzaldehyde $(1.0 \mathrm{mmol})$, ethyl acetoacetate $(1.2 \mathrm{mmol})$, urea $(1.5 \mathrm{mmol})$ in refluxing ethanol $(3.0 \mathrm{~mL})$ under ultrasonic irradiation.

Table 3. Calix[8]arene Sulfonic Acid Catalyzed Synthesis of 3,4-Dihydropyrimidin-2(1H)-ones 4a-p under Optimized Conditions ${ }^{a)}$

\begin{tabular}{|c|c|c|c|c|c|c|c|}
\hline \multirow{2}{*}{ Entry } & \multirow{2}{*}{ Product } & \multirow{2}{*}{$\mathrm{R}$} & \multirow{2}{*}{$\mathrm{X}$} & \multirow{2}{*}{ Time (min) } & \multirow{2}{*}{ Yield (\%) } & \multicolumn{2}{|c|}{ Melting point $\left({ }^{\circ} \mathrm{C}\right)$} \\
\hline & & & & & & Found & Reported \\
\hline 1 & $4 a$ & 4- $\mathrm{Cl}-\mathrm{C}_{6} \mathrm{H}_{4}$ & $\mathrm{O}$ & 20 & 90 & $215-216$ & $210-212^{27)}$ \\
\hline 2 & $4 b$ & 4- $\mathrm{CH}_{3}-\mathrm{C}_{6} \mathrm{H}_{4}$ & $\mathrm{O}$ & 22 & 91 & $216-217$ & $214-215^{9)}$ \\
\hline 3 & $4 c$ & 4-t-Bu- $\mathrm{C}_{6} \mathrm{H}_{4}$ & $\mathrm{O}$ & 30 & 82 & $156-158$ & - \\
\hline 4 & 4d & $2-\mathrm{CH}_{3} \mathrm{O}-\mathrm{C}_{6} \mathrm{H}_{4}$ & $\mathrm{O}$ & 20 & 93 & $260-261$ & $258-259^{28)}$ \\
\hline 5 & $4 e$ & $4-\mathrm{HO}-\mathrm{C}_{6} \mathrm{H}_{4}$ & $\mathrm{O}$ & 20 & 84 & $224-225$ & $226-227^{10)}$ \\
\hline 6 & $4 f$ & $4-\mathrm{F}-\mathrm{C}_{6} \mathrm{H}_{4}$ & $\mathrm{O}$ & 20 & 86 & $179-181$ & $173-176^{27)}$ \\
\hline 7 & $4 \mathrm{~g}$ & $3-\mathrm{CH}_{3}-\mathrm{C}_{6} \mathrm{H}_{4}$ & $\mathrm{O}$ & 25 & 88 & $228-229$ & $228-230^{28)}$ \\
\hline 8 & $4 h$ & $4-\mathrm{Br}-\mathrm{C}_{6} \mathrm{H}_{4}$ & $\mathrm{O}$ & 20 & 84 & $214-215$ & $213-215^{29)}$ \\
\hline 9 & $4 i$ & $3-\mathrm{NO}_{2}-\mathrm{C}_{6} \mathrm{H}_{4}$ & $\mathrm{O}$ & 40 & 68 & $231-233$ & $228-230^{29)}$ \\
\hline 10 & $4 j$ & $4-\mathrm{NO}_{2}-\mathrm{C}_{6} \mathrm{H}_{4}$ & $\mathrm{O}$ & 40 & 67 & $210-212$ & $211-213^{29)}$ \\
\hline 11 & $4 k$ & $\mathrm{CH}_{2} \mathrm{CH}_{3}$ & $\mathrm{O}$ & 48 & 46 & $174-175$ & $178-180^{29)}$ \\
\hline 12 & 41 & $\mathrm{CH}_{2} \mathrm{CH}_{2} \mathrm{CH}_{3}$ & $\mathrm{O}$ & 45 & 57 & $176-178$ & $180-182^{30)}$ \\
\hline 13 & $4 m$ & $\mathrm{C}_{6} \mathrm{H}_{5}-\mathrm{CH}=\mathrm{CH}$ & $\mathrm{O}$ & 35 & 76 & $232-233$ & $231-234^{30)}$ \\
\hline 14 & $4 n$ & $\mathrm{C}_{6} \mathrm{H}_{5}$ & $\mathrm{~S}$ & 20 & 92 & $207-209$ & $206-208^{10)}$ \\
\hline 15 & 40 & 4- $\mathrm{CH}_{3} \mathrm{O}-\mathrm{C}_{6} \mathrm{H}_{4}$ & $\mathrm{~S}$ & 20 & 90 & $150-151$ & $151-152^{10)}$ \\
\hline 16 & $4 p$ & $\mathrm{C}_{6} \mathrm{H}_{5}-\mathrm{CH}=\mathrm{CH}$ & $\mathrm{S}$ & 30 & 75 & $241-242$ & $243-245^{31)}$ \\
\hline
\end{tabular}

a) Reaction conditions: aromatic aldehydes $(1.0 \mathrm{mmol})$, ethyl acetoacetate $(1.0 \mathrm{mmol})$, urea $(1.5 \mathrm{mmol})$, calix $[8]$ arene sulfonic acid $(0.2 \mathrm{~mol} \%)$, in refluxing ethanol $(3.0 \mathrm{~mL})$ under ultrasonic irradiation. 


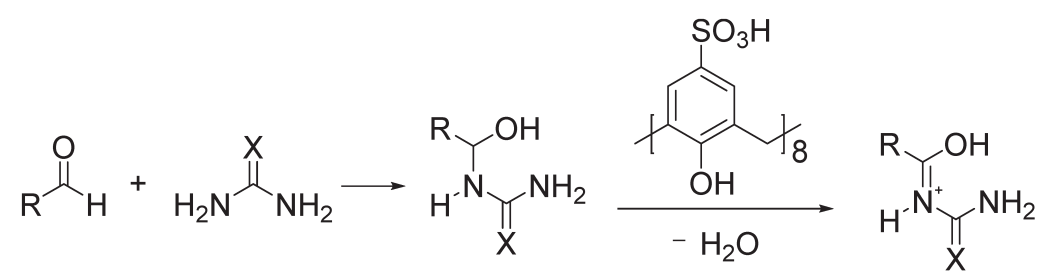

(A)

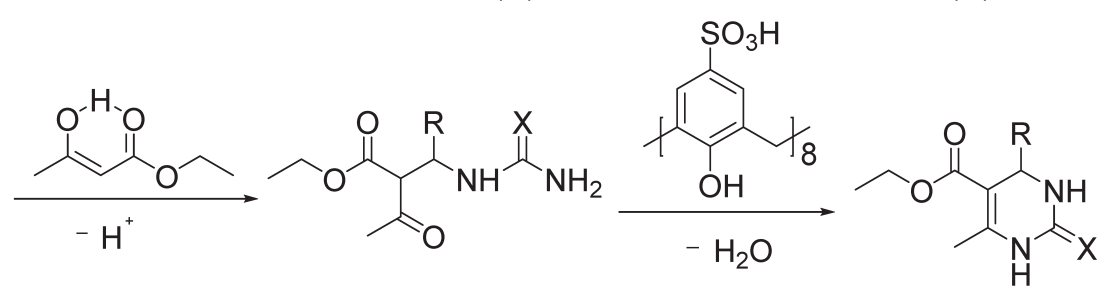

(C)

Chart 2. The Mechanism of Biginelli Reactions Catalyzed by Calix[8]arene Sulfonic Acid

acid. The results showed that no desired product was afforded in the absence of catalyst. When $0.1 \mathrm{~mol} \%$ calix[8]arene sulfonic acid was selected to catalyze the model reaction, the desired 3,4-dihydropyrimidine $4 \mathbf{a}$ was obtained in 35\% yield. When the catalyst amount was increased to $0.2 \mathrm{~mol} \%$, the yield improved by $90 \%$. However, large amount of catalyst $(1.0 \mathrm{~mol} \%)$ did not significantly improve the yield. This behavior is explained by the changes in polarity and $\mathrm{pH}$ of the reaction system. Thus, $0.2 \mathrm{~mol} \%$ calix[8]arene sulfonic acid was selected for all the reactions.

The same model reaction in the presence of $0.2 \mathrm{~mol} \%$ calix [8]arene sulfonic acid was carried out using different molar ratio of reagents. The best results were obtained with a $1: 1: 1.5$ ratio of 4-cholorobenzaldehyde to ethyl acetoacetate and urea.

Encouraged by the results obtained from the model reaction, the methodology was extended to a variety of aldehydes (aromatic, aliphatic, $\alpha, \beta$-unsaturated) with ethyl acetoacetate and urea in the presence of $0.2 \mathrm{~mol} \%$ calix[8]arene sulfonic acid for the condensation reaction to give the products $4 \mathbf{a}-\mathbf{m}$. Thiourea has been used with similar success to provide corresponding 3,4-dihydropyrimidine-2(1H)-thiones $\mathbf{4 n - p}$. In Table 3 , the reaction proceeded smoothly with aromatic aldehydes carrying either electron-donating or electron-withdrawing groups in the ortho-, meta-, and para-positions in moderate to high yields of $67-93 \%$. However, the condensation reaction of aliphatic aldehydes (entries $\mathbf{4 k}, \mathbf{4 l}$ ) exhibited lower yields (of 46-57\%) and longer reaction time compared with aromatic aldehydes, which was due to the decomposition or polymerization of aliphatic aldehydes under acidic conditions.

On the basis of the experimental results and reported acid catalyzed Biginelli reactions, ${ }^{7,8,10)}$ a plausible mechanism has been concisely shown in Chart 2. Firstly, the nucleophilic addition of urea or thiourea to aldehyde gives adduct (A). Subsequently, an enol to acyl iminium intermediate (B) is formed by dehydration of acyl imine catalyzed by calix[8]arene sulfonic acid. Further condensation of iminium ion (B) with ethyl acetoacetate, presumably through its enol tautomer affords the intermediate (C), followed by the final acid-catalyzed cyclization of the intermediate $(\mathbf{C})$ with the elimination of water to produce the corresponding 3,4-dihydropyrimidin-2 $(1 H)$-one/ thione. In this process, calix[8]arene sulfonic acid exerts an important function on assisting the accomplishment of several steps mostly due to its strong hydrogen bonding ability.

\section{CONCLUSION}

In summary, we successfully developed a simple and efficient method for the synthesis of dihydropyrimidiones/thiones by using calix[8]arene sulfonic acid to catalyze three-component reaction under ultrasonic irradiation. The advantages of the reactions contain not only the easy isolated procedure, short reaction time and the catalyst's very low cost, but also fewer byproducts. In addition to this, this work also provides a new example of potential application of functionalized calixarenes in organic synthesis.

Acknowledgments This work was supported by the National Natural Science Foundation of China (No. 81202490); the Natural Science Foundation of the Jiangsu Higher Education Institutions of China (No. 12KJB350005); the Innovative Practice Training Program for Undergraduates of Jiangsu Higher Education Institutions (No. 201310313006Z); the National Training Program of Innovation and Entrepreneurship for Undergraduates (No. 201310313006).

Conflict of Interest The authors declare no conflict of interest.

\section{REFERENCES}

1) Kappe CO. A review on DHPMs. Tetrahedron, 49, 6937-6963 (1993).

2) Kappe CO. Biologically active dihydropyrimidones of the Biginellitype-a literature survey. Eur. J. Med. Chem., 35, 1043-1052 (2000).

3) Atwal KS, Swanson BN, Unger SE, Floyd DM, Moreland S, Hedberg A, O'Reilly BC. Dihydropyrimidine calcium channel blockers. 3-Carbamoyl-4-aryl-1,2,3,4-tetrahydro-6-methyl-5-pyrimidinecarboxylic acid esters as orally effective antihypertensive agents. J. Med. Chem., 34, 806-811 (1991).

4) Kappe CO, Fabian WMF, Semones MA. Conformational analysis of 4-aryl-dihydropyrimidine calcium channel modulators. A comparison of $a b$ initio, semiempirical and X-ray crystallographic studies. Tetrahedron, 53, 2803-2816 (1997). 
5) Atwal KS, Rovnyak GC, O’Reilly BC, Schwartz J. Substituted 1,4-dihydropyrimidines. 3. Synthesis of selectively functionalized 2-hetero-1,4-dihydropyrimidines. J. Org. Chem., 54, 5898-5907 (1989).

6) Mayer TU, Kapoor TM, Haggarty SJ, King RW, Schreiber SL, Mitchison TJ. Small molecule inhibitor of mitotic spindle bipolarity identified in a phenotype-based screen. Science, 286, 971-974 (1999).

7) Kuarm BS, Madhav JV, Laxmi SV, Rajitha B. Xanthan Sulfuric Acid: A new and efficient biosupported solid acid catalyst for the synthesis of 3,4-dihydropyrimidin-2(1H)-ones. Synth. Commun., 42, 1211-1217 (2012).

8) Saloutin VI, Burgart YV, Kuzueva OG, Kappe CO, Chupakhin ON. Biginelli condensations of fluorinated 3-oxo esters and 1,3-diketones. J. Fluor. Chem., 103, 17-23 (2000).

9) $\mathrm{Hu} \mathrm{EH}$, Sidler DR, Dolling UH. Unprecedented catalytic three component one-pot condensation reaction: an efficient synthesis of 5-alkoxycarbonyl-4-aryl-3,4-dihydropyrimidin-2(1H)-ones. J. Org. Chem., 63, 3454-3457 (1998).

10) Rahmatpour A. Polyvinylsulfonic acid: An efficient, water-soluble and reusable Brønsted acid catalyst for the three-component synthesis of 3,4-dihydropyrimidin-2(1H)-ones/thiones in water and ethanol. Catal. Lett., 142, 1505-1511 (2012).

11) Narsaiah AV, Reddy AR, Yadav JS. Samarium triflate-catalyzed biginelli condensation: An improved method for the synthesis of 3,4-dihydropyrimidin-2(1H)-ones. Synth. Commun., 41, 2794-2799 (2011).

12) Cepanec I, Litvić M, Filipan-Litvić M, Grüngold I. Antimony(III) chloride-catalysed biginelli reaction: a versatile method for the synthesis of dihydropyrimidinones through a different reaction mechanism. Tetrahedron, 63, 11822-11827 (2007).

13) Fang D, Luo J, Zhou XL, Yang ZW, Liu ZL. One-pot green procedure for Biginelli reaction catalyzed by novel task-specific roomtemperature ionic liquids. J. Mol. Catal., 274, 208-211 (2007).

14) Liu CJ, Wang JD. Ultrasound-assisted synthesis of novel 4-(2phenyl-1,2, 3-triazol-4-yl)-3,4-dihydropyrimidin-2(1H)-(thio)ones catalyzed by $\mathrm{Sm}\left(\mathrm{ClO}_{4}\right)_{3}$. Molecules, 15, 2087-2095 (2010).

15) Panda SS, Khanna P, Khanna L. Biginelli reaction: a green perspective. Curr. Org. Chem., 16, 507-520 (2012).

16) Safari J, Zarnegar Z. Biginelli reaction on $\mathrm{Fe}_{3} \mathrm{O}_{4}-\mathrm{MWCNT}$ nanocomposite: excellent reactivity and facile recyclability of the catalyst combined with ultrasound irradiation. RSC Adv., 3, 1796217967 (2013)

17) Pagadala R, Maddila S, Jonnalagadda SB. Eco-efficient ultrasonic responsive synthesis of pyrimidines/pyridines. Ultrason. Sonochem., 21, 472-477 (2014).

18) An L, Zhang L, Zheng YG, Xue YS, Mou J, Liu L, Liu Y. Microwave irradiation assisted selective synthesis of 4,6-diaryl-3,4-dihy-
dropyrimidin-2(1H)-ones and pyrimidin-2(1H)-ones. Chin. J. Org. Chem., 32, 1108-1111 (2012).

19) Shimizu S, Shimada N, Sasaki Y. Mannich-type reactions in water using anionic water-soluble calixarenes as recoverable and reusable catalysts. Green Chem., 8, 608-614 (2006).

20) Liu YL, Liu L, Wang YL, Han YC, Wang D, Chen YJ. Calix[n]arene sulfonic acids bearing pendant aliphatic chains as recyclable surfactant-type Brønsted acid catalysts for allylic alkylation with allyl alcohols in water. Green Chem., 10, 635-640 (2008).

21) Fernandes SA, Natalino R, Gazolla PAR, Silva MJ, Jham GN. $p$-Sulfonic acid calix[n]arenes as homogeneous and recyclable organocatalysts for esterification reactions. Tetrahedron Lett., 53, 1630-1633 (2012).

22) Sayin S, Yilmaz M. Brønsted acidic magnetic nano- $\mathrm{Fe}_{3} \mathrm{O}_{4}$-adorned calix $[n]$ arene sulfonic acids: synthesis and application in the nucleophilic substitution of alcohols. Tetrahedron, 70, 6669-6676 (2014).

23) Baghbanian SM, Babajani Y, Tashakorian H, Khaksar S, Farhang M. $p$-Sulfonic acid calix[4]arene: An efficient reusable organocatalyst for the synthesis of bis(indolyl)methanes derivatives in water and under solvent-free conditions. C. R. Chim., 16, 129-134 (2013).

24) Silva DL, Fernandes SA, Sabino AA, Fatima A. $p$-Sulfonic acid calixarenes as efficient and reusable organocatalysts for the synthesis of 3,4-dihydropyrimidin-2(1H)-ones/thiones. Tetrahedron Lett., 52, 6328-6330 (2011).

25) An L, Zhu X, Zhang JH, Zhu HD, Zhu J. Efficient synthesis of $p$-sulfonic acid calixarene under ultrasonic irradiation. Chemical Reagents, 36, 755-757 (2014).

26) Zhidovinova MS, Fedorova OV, Rusinov GL, Ovchinnikova IG. Multicomponent sonochemical synthesis of podands. Mol. Divers., 6, 323-326 (2003).

27) Reddy CV, Mahesh M, Raju PVK, Babu TR, Reddy VVN. Zirconium(IV) chloride catalyzed one-pot synthesis of 3,4-dihydropyrimidin-2(1H)-ones. Tetrahedron Lett., 43, 2657-2659 (2002).

28) Boumoud T, Boumoud B, Rhouati S, Belfaitah A. An efficient and recycling catalyst for the one-pot three-component synthesis of substituted 3,4-dihydropyrimidin-2(1H)-ones. J. Chem., 5, 688-695 (2008).

29) Russowsky D, Benvenutti EV, Roxo GS, Grasel F. Multicomponent synthesis of 3,4-dihydropyrimidin-2-(1H)-ones with a $\mathrm{Cu} /$ silica $\mathrm{xe}-$ rogel composite catalyst. Lett. Org. Chem., 4, 39-42 (2007).

30) Nasr-Esfahani M, Hoseini SJ, Mohammadi $\mathrm{F}$. $\mathrm{Fe}_{3} \mathrm{O}_{4}$ nanoparticles as an efficient and magnetically recoverable catalyst for the synthesis of 3,4-dihydropyrimidin-2(1H)-ones under solvent-free conditions. Chin. J. Catal., 32, 1484-1489 (2011).

31) Liu CJ, Wang J, Li YP. One-pot synthesis of 3,4-dihydropyrimidin$2(1 \mathrm{H})$-(thio)ones using strontium(II) nitrate as a catalyst. J. Mol. Catal. A, 258, 367-370 (2006). 